\title{
All-optical wavelength conversion and signal regeneration using an electroabsorption modulator
}

\author{
Højfeldt, Sune; Bischoff, Svend; Mørk, Jesper
}

Published in:

Journal of Lightwave Technology

Link to article, DOI:

$10.1109 / 50.857758$

Publication date:

2000

Document Version

Publisher's PDF, also known as Version of record

Link back to DTU Orbit

Citation (APA):

Højfeldt, S., Bischoff, S., \& Mørk, J. (2000). All-optical wavelength conversion and signal regeneration using an electroabsorption modulator. Journal of Lightwave Technology, 18(8), 1121-1127.

https://doi.org/10.1109/50.857758

\section{General rights}

Copyright and moral rights for the publications made accessible in the public portal are retained by the authors and/or other copyright owners and it is a condition of accessing publications that users recognise and abide by the legal requirements associated with these rights.

- Users may download and print one copy of any publication from the public portal for the purpose of private study or research.

- You may not further distribute the material or use it for any profit-making activity or commercial gain

- You may freely distribute the URL identifying the publication in the public portal

If you believe that this document breaches copyright please contact us providing details, and we will remove access to the work immediately and investigate your claim. 


\title{
All-Optical Wavelength Conversion and Signal Regeneration Using an Electroabsorption Modulator
}

\author{
Sune Højfeldt, Svend Bischoff, and Jesper Mørk
}

\begin{abstract}
All-optical wavelength conversion and signal regeneration based on cross-absorption modulation in an InGaAsP quantum well electroabsorption modulator (EAM) is studied at different bit rates. We present theoretical results showing wavelength conversion efficiency in agreement with existing experimental results, and the signal regeneration capability of the device is investigated. In particular, we demonstrate the dependence of the extinction ratio of both the converted signal and the control signal on the device length and on the power level of the control signal. We also show how the sweep-out dynamics influences the results.
\end{abstract}

Index Terms-All-optical wavelength conversion, electroabsorption modulator (EAM), optical communication, semiconductor devices, signal regeneration, ultrafast signal processing.

\section{INTRODUCTION}

I $\mathrm{N}$ the last decade, the electroabsorption modulator (EAM) has found a wider and wider range of applications within optical communications, although so far mostly at the research level. Around the early $1990 \mathrm{~s}$, functionalities such as pulse generation [1]-[4] (which has now found commercial application in integrated distributed feedback laser/EAM modules), demultiplexing [5] and signal regeneration [6] were demonstrated using an EAM. These functionalities are important for constructing ultrahigh-speed all-optical networks [7].

With the quite recent achievements of fast demultiplexing from 100 to $10 \mathrm{~Gb} / \mathrm{s}$ [8], widely tunable pulse generation using an EAM [9], all-optical wavelength conversion and signal regeneration [10], [11], the usefulness of the EAM as an integrated component for signal processing has been further substantiated. The EAM is also interesting because its range of operation is quite large, as can be seen in, e.g., [6] where an on-off ratio of $20 \mathrm{~dB}$ is obtained from 1.53 to $1.57 \mu \mathrm{m}$ with reverse biases less than $5 \mathrm{~V}$.

The above applications show that the EAM is capable of providing high on-off ratios, either by electrical modulation of the absorption (electroabsorption) or by optical means (cross-absorption modulation), which includes all absorption changes induced by photo-generated carriers.

In this paper, we focus on the all-optical properties of the EAM where a fixed dc bias is applied to adjust the band edge as well as for ensuring a fast sweep-out of the photogenerated carriers. All-optical wavelength conversion up to $40 \mathrm{~Gb} / \mathrm{s}$

Manuscript received December 7, 1999; revised May 2, 2000. This work was supported by the Danish Technical Research Council (STVF) through the SCOOP program.

The authors are with Research Center COM, Technical University of Denmark, Lyngby DK-2800, Denmark (e-mail: sh@com.dtu.dk).

Publisher Item Identifier S 0733-8724(00)06476-8. was recently carried out, with good performance up to 20 $\mathrm{Gb} / \mathrm{s}$ [10]-[12]. The all-optical wavelength conversion in an EAM relies on the saturable absorption characteristic, through cross-absorption modulation (XAM). Note that this method avoids the signal inversion which results when using cross-gain modulation (XGM) in semiconductor optical amplifiers [13]. Native signal regeneration (where the incoming signal itself is improved and not converted to a new, locally generated signal) at $10 \mathrm{~Gb} / \mathrm{s}$ was also demonstrated [11], [12]. The regenerative capability of the EAM relies on saturation of the absorption by the incoming signal itself.

We present theoretical results that are in good agreement with available experimental results, and investigate the influence of device length and input power level of the control signal on the conversion and regeneration results. We also show the influence of the sweep-out dynamics on the quality of the results.

The paper is organized as follows: In Section II we describe the model used in the simulations and the configuration used for performing the wavelength conversion and signal regeneration. In Section III the simulation results are presented. First, the nonlinearity in the absorption as function of input pulse energy is described. We then describe wavelength conversion and signal regeneration at 10,20, and $40 \mathrm{~Gb} / \mathrm{s}$, and show the influence of various parameters on the results. Finally, Section IV summarizes and concludes the paper.

\section{Model AND SETUP}

The model used for the reverse-biased quantum well absorber is a large-signal model originally developed for studying colliding-pulse mode-locked lasers [14]. The model includes propagation effects, and is based on a detailed gain model, derived using the density matrix formalism. Ultrafast effects such as spectral hole-burning and carrier heating are taken into account, while excitonic effects are not included in the model. We consider a device with five quantum wells, and restrict our attention to transverse electric (TE) polarized light.

Further, a simple carrier density dependent sweep-out time is assumed. In the literature, sweep-out times on the order of several tens of picoseconds in multiple quantum well (MQW) InGaAsP and AlGaAs structures have been reported, even at relatively high reverse biases, around $\sim-5 \mathrm{~V}$ [15], [16]. Elsewhere, saturable absorbers have displayed faster recovery times, down to $8 \mathrm{ps}$ [11], [17]. We have implemented a simple model, where the sweep-out time varies as a function of the carrier density, from 8 ps at low densities to $25 \mathrm{ps}$ at transparency, based on results in [10]. The mechanism that leads to longer sweep-out times at higher carrier densities is screening of the applied field by photogenerated carriers, see, e.g., [15], [16]. 


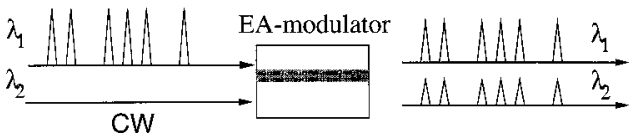

Fig. 1. The setup in the simulations. A data signal at wavelength $\lambda_{1}$ and a $\mathrm{CW}$ signal at $\lambda_{2}$ are injected into an EAM from the same end (copropagation scheme). Through XAM the information from the control signal at $\lambda_{1}$ is transferred to the $\mathrm{CW}$ signal at $\lambda_{2}$.

Fig. 1 shows a schematic of the wavelength conversion setup. A control signal at wavelength $\lambda_{1}$ containing a bit sequence, and a continuous-wave (CW) signal at $\lambda_{2}$ are launched into the EAM. In our simulations we have mainly considered the co-propagation scheme, in which the two signals enter the device from the same end as shown in Fig. 1. At the output end of the EAM, the bit-sequence represented by the control signal has been transferred to the $\mathrm{CW}$ signal through XAM.

\section{RESULTS}

In the following simulations, we investigate the absorption nonlinearity of our device, as well as the use of this nonlinearity for performing wavelength conversion and signal regeneration. In all the results presented in this paper, the pulse full-width at half-maximum (FWHM) is set to $8 \mathrm{ps}$.

For the remaining part of the paper, all power and energy levels refer to in-chip levels. The results of course depend on the confinement factor of the optical field in the chip.

\section{A. The Absorption Nonlinearity}

In Fig. 2, the calculated transmitted pulse energy is shown as a function of input pulse energy for various wavelengths corresponding to energies above the bandgap. For small pulse energies, the absorption corresponds to an essentially empty conduction band, and hence the absorption due to promotion of carriers to the conduction band is high. When the carrier density comes close to the transparency density (i.e., when the pulse energy is large), the stimulated absorption is very low and the total absorption is approximately equal to the total internal loss. The total internal loss is made up of losses in the active region (such as free-carrier absorption), absorption in the cladding, and scattering losses [18], and is in this paper taken to be $40 \mathrm{~cm}^{-1}$. The results are quite insensitive to the exact value of the internal loss because the total absorption is dominated by stimulated absorption.

Fig. 2 also indicates another important issue, namely that the output energy is severely reduced if one operates at shorter wavelengths. This is also expected, since the absorption is larger at shorter wavelengths. The saturation energy also increases: At shorter wavelengths the photogenerated (quasi-equilibrium) carrier density leads to a smaller change of the absorption. This means that saturation of the absorption at shorter wavelengths requires much more energetic pulses.

If one relies entirely on the change in absorption obtained through phase-space filling, it is not desirable to have $\lambda_{2}$ (the $\mathrm{CW}$ signal wavelength) at the longer wavelengths (close to the band edge). This is because the change in absorption here is relatively small, due to the low density of states. On the other hand, the absorption increases at shorter wavelengths, resulting

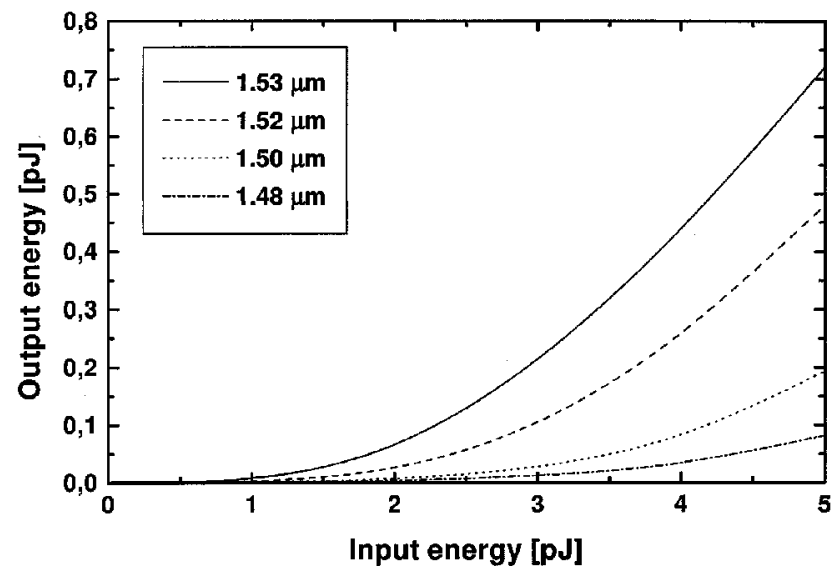

Fig. 2. The graph show the output pulse energy as function of input pulse energy. The pulses propagate through a $150-\mu \mathrm{m}$-long device. The results are shown for four different wavelengths, and illustrate the nonlinear absorption property of the EAM.

in a reduced output power. Hence, a compromise must be made to obtain both a reasonable output power (pulling to the longer wavelength side), and an efficient modulation of the carrier density (pulling to the shorter wavelength side).

By the same token, the controlling signal at $\lambda_{1}$ should not be too close to the band edge, or its overall influence on the carrier density will be small. Its influence on the absorption at the CW wavelength $\lambda_{2}$ will correspondingly be small. If one wishes to regenerate the signal at $\lambda_{1}$, this wavelength should not be too far into the band, again since the output power decreases with decreasing wavelength.

\section{B. Wavelength Conversion and Signal Regeneration at $10 \mathrm{~Gb} / \mathrm{s}$}

To demonstrate the wavelength conversion and signal regeneration capability of the EAM, we propagate various bit patterns consisting of 8-ps wide return-to-zero (RZ) pulses with an extinction ratio (ER) of $10 \mathrm{~dB}$ (peak-to-floor) through the device. The bit-patterns used were chosen such that the worst possible eye was obtained. The 10-dB extinction ratio (ER) is introduced by adding a constant background to the bit sequence at the wavelength $\lambda_{1}$. The background allows us to simulate the presence of unwanted signals. This could for instance be an optical time-division multiplexing (OTDM) channel that was not perfectly cleared, or it could be spontaneous emission added by, e.g., signal generators and amplifiers (and other active components) previously encountered in the system.

Fig. 3 shows a simulation where a $10-\mathrm{dBm} \mathrm{CW}$ signal ("CW" in Fig. 3) at $\lambda_{2}=1520 \mathrm{~nm}$ and an 11.5-dBm average pulse power bit-stream signal ("signal" in Fig. 3) at $\lambda_{1}=1510 \mathrm{~nm}$ are launched into the EAM from the same end (copropagation scheme, cf., Fig. 1). Note that the quoted average pulse power includes the contribution from the background, which is relatively more important at low bit rates, where the duty-cycle is low, than at higher bit rates. In practice, the two signals would be separated at the output of the device using a spectral filter. The figure shows the power levels for mark and space as a function of device length. For increasing device length, the ER increases both for the control signal and for the converted signal 


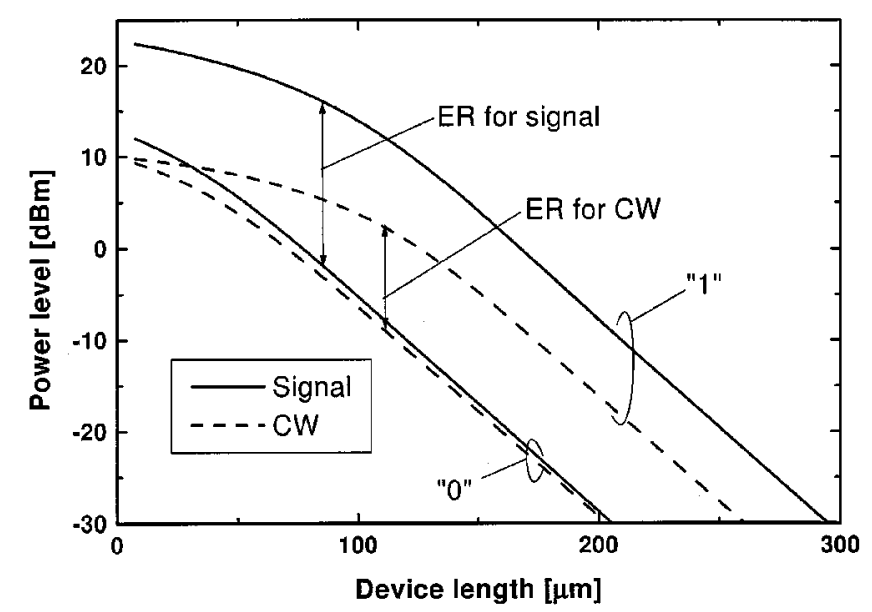

Fig. 3. Power levels for space ("0") and mark (" 1 ") as function of length for then control signal ("signal") and the CW signal ("CW"). The bit rate is $10 \mathrm{~Gb} / \mathrm{s}$. The input power of the control signal was $11.5 \mathrm{dBm}$, while the power in the $\mathrm{CW}$ signal was $10 \mathrm{dBm}$. The ER was extracted from worst-case eye-diagrams.

(the latter with an ER which is "0" at the input), demonstrating the wavelength conversion and signal regeneration capabilities of the device. When the control signal power level becomes too low to significantly influence the absorption (through the carrier density), the ER values become constant. The power level, however, keeps decreasing due to the internal loss and stimulated absorption.

Fig. 3 clearly shows that the improvement in ER comes about due to the nonlinear saturation properties: The energy in a " 0 " decreases more rapidly than the energy in a " 1 " due to the higher absorption for smaller input energy levels. To ensure a certain power level at the output (e.g., $10 \mathrm{~dB}$ above the amplified spontaneous emission (ASE) level in an amplifier), the length of the device must be kept below a certain value, which of course depends on the input power levels.

\section{Pulse Energy Dependence}

The obtainable ER depends on the input power level of the control signal. Fig. 4 shows the ER of a converted $10-\mathrm{Gb} / \mathrm{s}$ signal as a function of device length for various average control pulse energies. Corresponding for example to an average power of $11.5 \mathrm{dBm}$ (average pulse energy of $2.8 \mathrm{pJ}$ ), an ER of $\approx 12$ can be obtained on the converted signal. Fig. 4 also shows that the ER obtained below, say $150 \mu \mathrm{m}$, for the highest input power level shown (11.2 $\mathrm{pJ}$ average pulse energy) is smaller than that obtained using a range of lower input power levels. This is because the saturation of the absorption in the first part of the device suppresses efficient carrier density modulation, and hence mark and space will experience almost the same absorption. This indicates that for a given device length there is an optimum input power level giving the highest ER at the output. This is demonstrated in Fig. 5, where the ER at the output of a $110-\mu \mathrm{m}$-long device is shown as a function of the average control pulse energy. A maximum ER at the output is found in this case around an average pulse energy of $4 \mathrm{pJ}$. For a longer device, the maximum ER increases, and the peak occurs at a higher input energy.

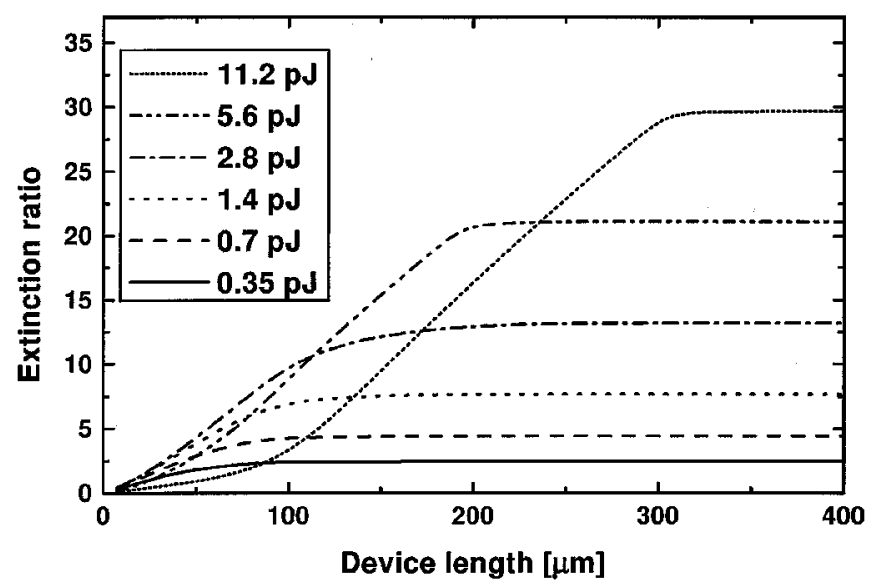

Fig. 4. ER of a converted 10-Gb/s signal as function of length with average control pulse energy as a parameter. The ER was extracted from worst-case eye-diagrams. The $\mathrm{CW}$ power level was $P_{\mathrm{CW}}=10 \mathrm{dBm}$.

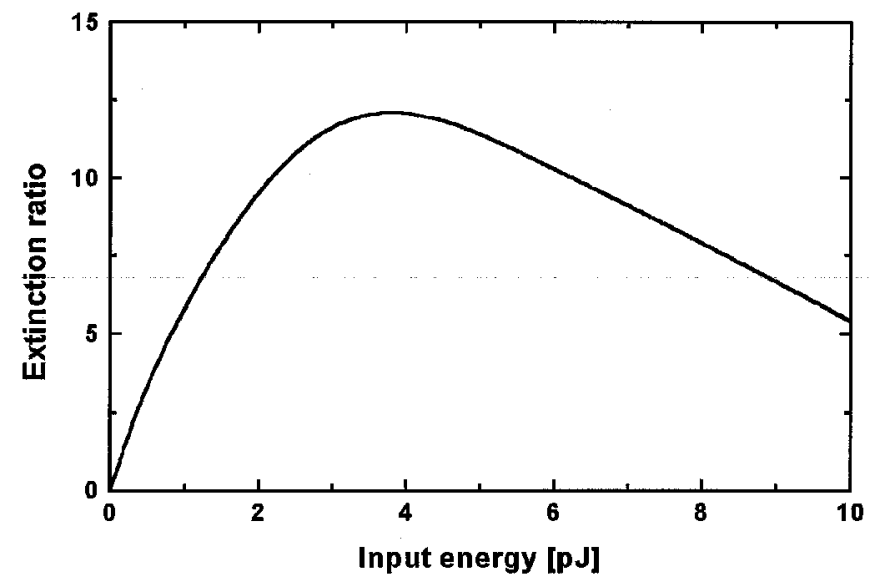

Fig. 5. Extinction ratio for the converted signal as function of average input energy of the control signal for a 110- $\mu \mathrm{m}$-long device. The bit rate was $10 \mathrm{~Gb} / \mathrm{s}$. The ER was extracted from worst-case eye-diagrams.

\section{Wavelength Conversion up to $40 \mathrm{~Gb} / \mathrm{s}$}

To investigate the wavelength conversion capability at higher bit rates, we modeled the propagation of 20 and $40 \mathrm{~Gb} / \mathrm{s}$ pulsetrains through the EAM. Again, we chose pulse-trains that ensured the worst possible eye-distortion.

To facilitate the understanding of the effects that are important, we display the simulated eye diagrams. Note that the diagrams include the background introduced in Section III-B, resulting in the before mentioned input extinction ratio of $10 \mathrm{~dB}$. We assumed that fixed and well-defined power levels entered the device. This is of course an idealization, and any noise on the input signals (amplitude or timing jitter) will reduce the quality of both the converted signal and the control signal. However, these are small effects compared to the distortions due to patterning, that we find even without noise. Noise on the input will tend to smear out the rather discrete levels found in the figures.

Eye-diagrams for different EAM lengths are shown for different power levels in Figs. 6 and 7. The control signal power 


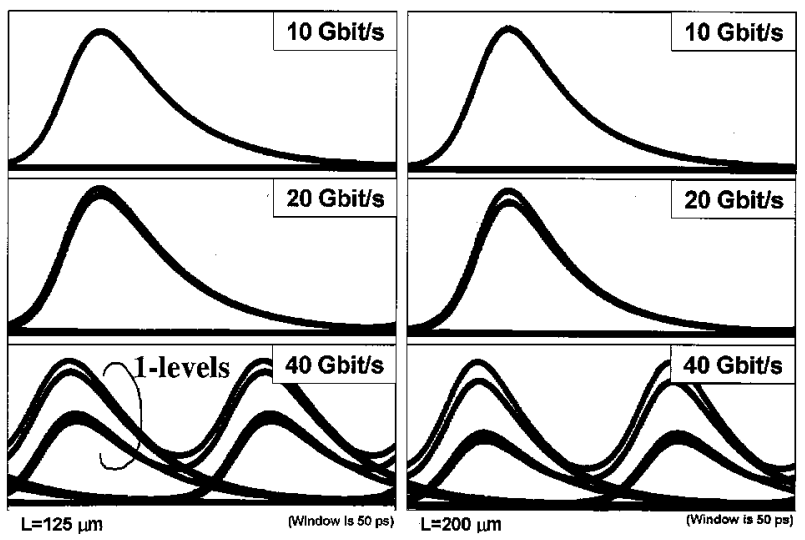

Fig. 6. Converted 10,20 , and $40 \mathrm{~Gb} / \mathrm{s}$ signal at the output of a $125-\mu \mathrm{m}$ long device (left) and a $200-\mu \mathrm{m}$ long device (right). The CW power was $10 \mathrm{dBm}$ and the signal power power is $11 \mathrm{dBm}$ at $10 \mathrm{~Gb} / \mathrm{s}, 16 \mathrm{dBm}$ at $20 \mathrm{~Gb} / \mathrm{s}$, and $19 \mathrm{dBm}$ at $40 \mathrm{~Gb} / \mathrm{s}$. We explicitly point out that the levels indicated in the left figure (and similar levels in the following figures) are all marks. The temporal window is $50 \mathrm{ps}$ for all bit rates.

levels for the 10, 20, and $40 \mathrm{~Gb} / \mathrm{s}$ signals in Fig. 6 were 13, 16, and $19 \mathrm{dBm}$, respectively, and 16,19, and $22 \mathrm{dBm}$, respectively, in Fig. 7. The results are shown for 125 and $200-\mu \mathrm{m}$ long devices.

In Fig. 6, the quality of the eye for the $10 \mathrm{~Gb} / \mathrm{s}$ signal hardly improves from 125 to $200 \mu \mathrm{m}$, because the remaining power level at $L=125 \mu \mathrm{m}$ is too small to change significantly the absorption (see also Fig. 3). Note that the width of the pulses making up the converted signal decreases slightly with length. The eye-quality in Fig. 6 for the $20 \mathrm{~Gb} / \mathrm{s}$ signal also hardly improves as the length of the EAM is increased, although some amplitude variation now occurs in the marks. This variation comes about because the available time per bit is short enough that a total recovery of the absorption is not achieved between bits. Finally, in the $40 \mathrm{~Gb} / \mathrm{s}$ case the converted signal eye for the 125 $\mu \mathrm{m}$ long device shows a large range of both the space and the mark levels (only the mark levels can be clearly distinguished in the diagrams), resulting again because the absorption is not allowed to recover from one bit to the next. As expected, the distortion is more severe at $40 \mathrm{~Gb} / \mathrm{s}$ compared to $20 \mathrm{~Gb} / \mathrm{s}$. The distortion seen in the eye agrees well with the experimental results in [10].

In Fig. 7, the power level for the control signal at each individual bit rate is twice as large as in Fig. 6 . At $10 \mathrm{~Gb} / \mathrm{s}$, this results in a better ER at both $125 \mu \mathrm{m}$ and $200 \mu \mathrm{m}$. This is a manifestation of the same dependence that was also shown in Fig. 4 in terms of the ER, namely that a moderately higher power level can result in a better ER at the output. At $20 \mathrm{~Gb} / \mathrm{s}$ there is some distortion, again because the absorption has not completely recovered from one bit to the next. The distortion especially at $L=200 \mu \mathrm{m}$ is larger than in the corresponding eye in Fig. 6 . The reason is that the higher power level for the control signal in Fig. 7 results in a longer sweep-out time. At the same time, however, the background is absorbed over a longer length due to the higher power level, resulting in a better ER. For the $40 \mathrm{~Gb} / \mathrm{s}$ case in Fig. 7, the distortion encountered in Fig. 6, where the mark splits up, is at first slightly suppressed because each pulse saturates the absorber more, but the background is also higher.

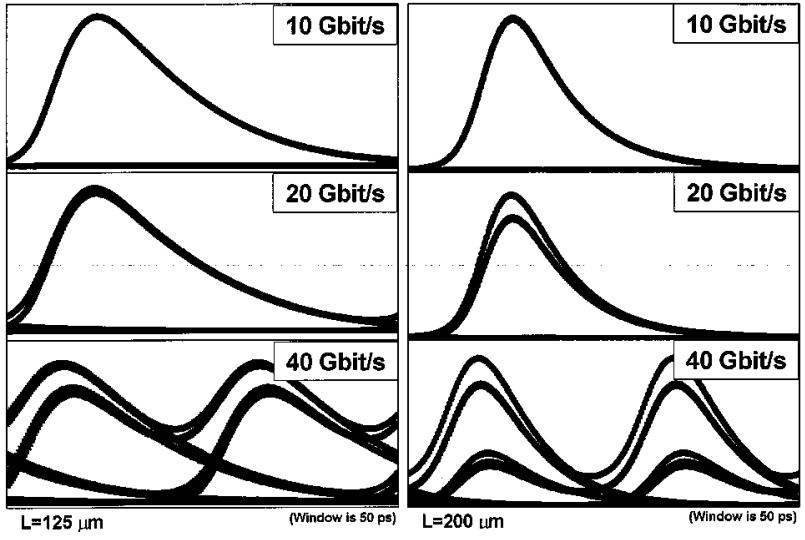

Fig. 7. Similar to Fig. 6, but the power level for control signal is $16 \mathrm{dBm}$ at $10 \mathrm{~Gb} / \mathrm{s}, 19 \mathrm{dBm}$ at $20 \mathrm{~Gb} / \mathrm{s}$, and $22 \mathrm{dBm}$ at $40 \mathrm{~Gb} / \mathrm{s}$. The temporal window is again 50 ps for all bit rates.

At $L=200 \mu \mathrm{m}$, the overall result is worsened compared to the corresponding eye in Fig. 6, just as for the $20 \mathrm{~Gb} / \mathrm{s}$ case.

Another interesting and important feature is seen when comparing for instance the 10-Gb/s converted signal in Fig. 6 with that of Fig. 7: The converted signal at the output of a $125 \mu \mathrm{m}$ long device is broader in Fig. 7 than in Fig. 6. However, at $L=200 \mu \mathrm{m}$, the situation is reversed. In general, this is due to the fact that the conversion happens over a longer distance for the more energetic signal. Initially the higher power level will give broader converted pulses due to a longer sweep-out time, but eventually (with length) the higher power levels give more narrow pulses due to the longer conversion length.

To summarize, the converted signal at $10 \mathrm{~Gb} / \mathrm{s}$ is improved by doubling the power level. For the 20-Gb/s signal, doubling the power gives a slightly worse ER for the shorter device, but for the longer device $(L=200 \mu \mathrm{m})$ the ER is improved and the width of the converted pulses is more narrow, but at the expense of some variation in the value of the converted marks. For the $40-\mathrm{Gb} / \mathrm{s}$ case, the higher power level initially helps to suppress amplitude variations, but lifts the background. For the longer device, the converted signal shows slight improvements in the ER for the higher power level, but as for the $20 \mathrm{~Gb} / \mathrm{s}$ case this happens at the expense of more amplitude variation in the mark, and even more so at $40 \mathrm{~Gb} / \mathrm{s}$ than at $20 \mathrm{~Gb} / \mathrm{s}$.

Note that the converted pulses thus generated are somewhat broader than the control pulses. This is a general property of this type of conversion scheme. It is especially the trailing edges of the converted pulses which are longer than those of the control pulses because the sweep-out dynamics "keeps the device open," thereby giving the converted pulses an asymmetric pulse shape. This asymmetry increases for longer sweep-out times, again demonstrating the importance of keeping the sweep-out time short.

\section{E. Signal Regeneration}

The nonlinearity used for performing wavelength conversion of course also allows a similar improvement in the ER for the control signal itself.

It has been shown that an incoming signal with a reminiscent pulse in the time-slot can be cleaned up [12]. We show in the 


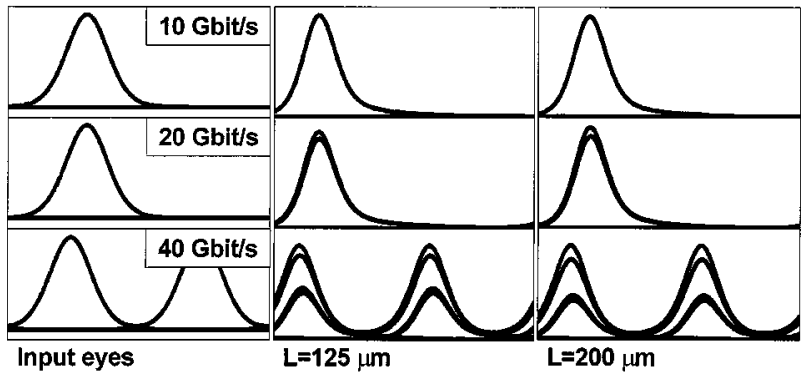

Fig. 8. Eye-diagrams for the control signal at the output of a $125 \mu \mathrm{m}$ long EAM (middle) and at the output of a $200 \mu \mathrm{m}$ long device (right). The input control signal is also shown (left). The eyes are shown for 10,20 , and $40 \mathrm{~Gb} / \mathrm{s}$ signals. The $\mathrm{CW}$ power was $10 \mathrm{dBm}$ and the signal power power is $13 \mathrm{dBm}$ at $10 \mathrm{~Gb} / \mathrm{s}, 16 \mathrm{dBm}$ at $20 \mathrm{~Gb} / \mathrm{s}$, and $19 \mathrm{dBm}$ at $40 \mathrm{~Gb} / \mathrm{s}$. The temporal window is $50 \mathrm{ps}$ for all bit rates.

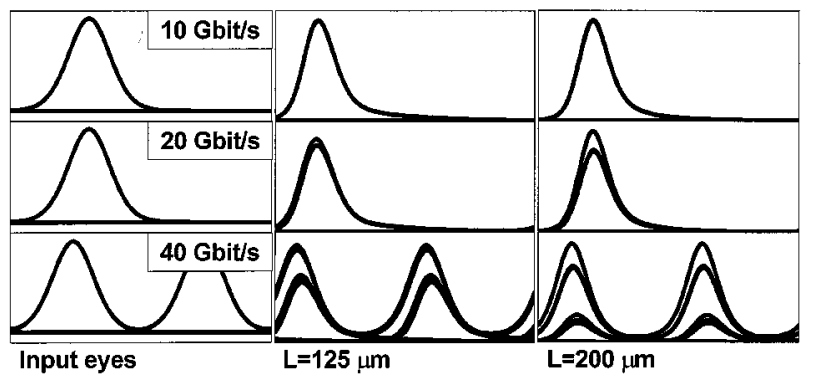

Fig. 9. Similar to Fig. 8, but the power level for control signal is $16 \mathrm{dBm}$ at $10 \mathrm{~Gb} / \mathrm{s}, 19 \mathrm{dBm}$ at $20 \mathrm{~Gb} / \mathrm{s}$, and $22 \mathrm{dBm}$ at $40 \mathrm{~Gb} / \mathrm{s}$. The temporal window is again $50 \mathrm{ps}$ for all bit rates.

following that the constant background introduced previously, with a power level of $10 \mathrm{~dB}$ less than the mark peak power can be suppressed substantially. This of course means that pulses with peak powers of $10 \mathrm{~dB}$ less than the actual marks can be suppressed at least as much.

Figs. 8 and 9 show the eyes for the control signal at the input and at the output of the device. The figures show features very similar to those found in the eye-diagrams for the converted signals (Figs. 6 and 7). The control signal is "born" with a 10-dB $\mathrm{ER}$, and hence the difference between the spaces and the marks at the output is much greater than for the converted signal. The mark variations are, however, almost identical to those of the converted signals. Note that there is a small compression of the pulse because the front of the pulse experiences a larger absorption than the rest of the pulse due to the finite sweep-out time.

One can see from the Figs. 8-9 only that there is an improvement in the ER but not the actual value of it. The ER for the eye-diagrams are therefore shown in Table I.

We see that quite a large improvement in the ER (which is 10 $\mathrm{dB}$ at the input) is obtained at $20 \mathrm{~Gb} / \mathrm{s}$, whereas the improvement for the $40 \mathrm{~Gb} / \mathrm{s}$ is seriously impaired because the absorption far from recovers from one bit to the next.

The mark variation found especially at $40 \mathrm{~Gb} / \mathrm{s}$ is of course unacceptable, and also the improvements obtained in the ERs are quite moderate for the device lengths shown. For the 10 and $20 \mathrm{~Gb} / \mathrm{s}$ signals, the impairment is small, but the improvements obtained are substantial. For instance a $20-\mathrm{Gb} / \mathrm{s}$ control signal with an average pulse power of $16 \mathrm{dBm}$ propagated through a
TABLE I

ER of 20-AND 40-Gb/s CONTROL Signals AT THE OUtPut of AN EAM. THE ER Is SHOWn FOR A $125-\mu \mathrm{m}$ AND A $200-\mu \mathrm{m}$-LONG DEVICE AND Two DifFerent Average INPUT Powers. The INITIAL ER Is $10 \mathrm{~dB}$. Numbers IN BRACKETS SHOW THE RESUlTS OBTAINED USING a CARRIER DENSITY INDEPENDENT SWEEP-OUT TIME OF $8 \mathrm{ps}$

\begin{tabular}{c|c|l|l}
\hline $\begin{array}{c}\text { Bit-rate } \\
(\mathrm{Gbit} / \mathrm{s})\end{array}$ & $\begin{array}{c}\text { Power } \\
(\mathrm{dBm})\end{array}$ & \multicolumn{1}{|c|}{$E R(\mathrm{~dB})$} & \multicolumn{1}{c}{$E R(\mathrm{~dB})$} \\
\hline 20 & 16 & 21.0 & 21.7 \\
& 19 & 23.4 & 29.5 \\
\hline \multirow{2}{*}{40} & 19 & $14.7(17.7)$ & $15.3 \quad(17.9)$ \\
& 22 & $14.8(20.9)$ & $17.3 \quad(23.3)$ \\
\hline
\end{tabular}

125 - $\mu \mathrm{m}$-long device is improved $\approx 11 \mathrm{~dB}$ from 10 to $21 \mathrm{~dB}$. Thus, the regenerative capability obtained here could potentially be useful if integrated, for instance. with a Mach-Zehnder interferometer used for wavelength conversion or drop functionalities. Interferometer switches based on XPM typically produce a finite background in the case of wavelength conversion, or small extraneous pulses in the case of the drop functionality. These effects occur due to imperfect phase matching during the gain recovery of the involved SOAs. These effects could be suppressed by integrating the interferometer with an EAM. The power level at the output of the interferometer is quite large because the SOAs involved are strongly biased to achieve high gain, and thus matches well the conditions we have found for producing significant improvements.

\section{F. Co- and Counterpropagation Schemes}

So far we have considered the copropagation scheme where the control and CW signals are launched into the same end of the device as shown in Fig. 1. However, the wavelength conversion can also be performed by launching the two signals into the device from opposite ends (counter-propagation scheme). The latter method allows for conversion to the same wavelength, and circumvents the need for spectral filtering at the output of the device.

Fig. 10 shows the ER of a converted 40-Gb/s signal obtained for various control signal power levels and $P_{\mathrm{CW}}=11 \mathrm{dBm}$. The figure shows that the difference between the ERs of the converted signals obtained with the two schemes increases with the control signal power level. This is expected for our device where the control pulses are wider than the transit time through the device. The difference between the two schemes is almost entirely caused by an increased absorption saturation in the copropagation scheme, where the signals are injected into the same end of the device. This results in a less efficient carrier modulation in the first part of the device.

We also performed the calculation with $P_{\mathrm{CW}}=-1 \mathrm{dBm}$, but found less than $0.4 \mathrm{~dB}$ difference between the two schemes for the signal power levels shown in Fig. 10, demonstrating again that the difference is due to the combined effects of a longer 


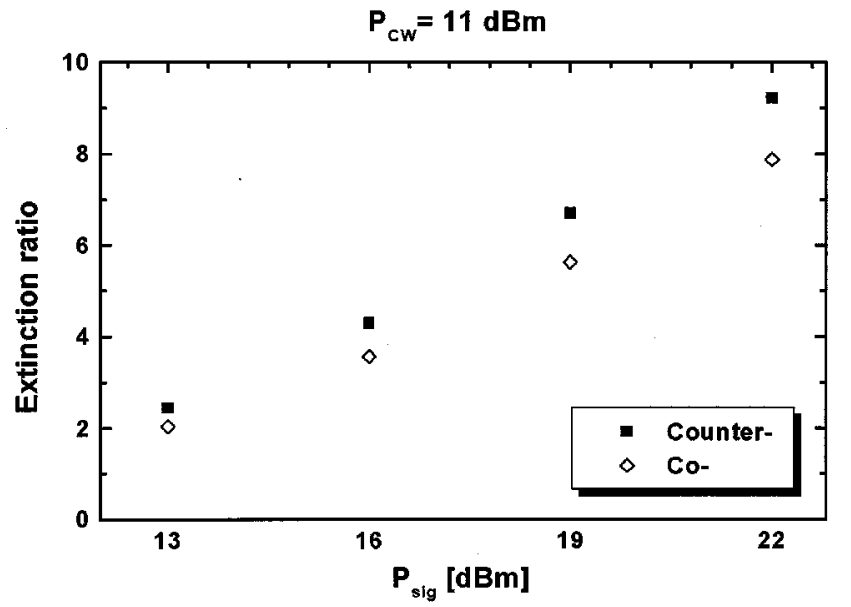

Fig. 10. The output ER of the converted signal as function of the input control signal power level. The input power level of the CW light is $11 \mathrm{dBm}$. The bit rate was $40 \mathrm{~Gb} / \mathrm{s}$. The device length was $200 \mu \mathrm{m}$.

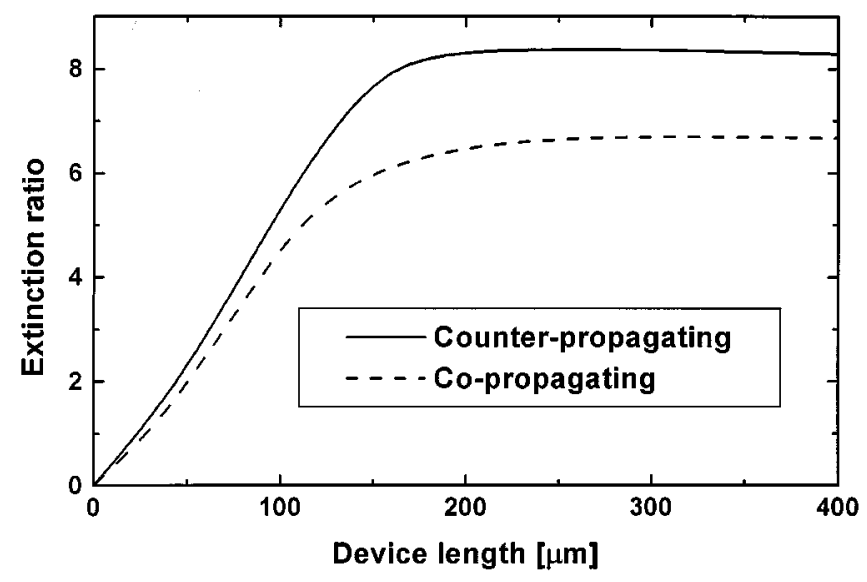

Fig. 11. Extinction ratio of a converted 10-Gb/s signal as function of device length for co- and counterpropagation schemes. The $\mathrm{CW}$ power was $15 \mathrm{dBm}$, and the control signal average pulse power was $8 \mathrm{dBm}$.

sweep-out time and smaller differential gain resulting when both signals enter the device from the same end.

Fig. 11 shows the ER of a converted 10-Gb/s signal as a function of length for the two different schemes with $P_{\mathrm{CW}}=15$ $\mathrm{dBm}$ and an average control pulse power $P_{\mathrm{sig}}=8 \mathrm{dBm}$. As expected, both level out as the remaining power decreases below the saturation power. However, the counter-propagation scheme has a better overall performance in this case.

The conclusions in this section are likely modified when one considers short pulses, where the width of the pulse is small compared to the transit time through the device. In this case, the overlap between the control pulse and the $\mathrm{CW}$ signal will likely favor the copropagation scheme.

\section{G. The Importance of Reducing the Sweep-Out Time}

To demonstrate the effect that the field screening (caused by photogenerated carriers) have on the results, Fig. 12 shows the signal regeneration at $40 \mathrm{~Gb} / \mathrm{s}$ for two cases: In the first case, the sweep-out time varies between 8 and $25 \mathrm{ps}$ (used in the previous part of the paper and further described back in Section II). In

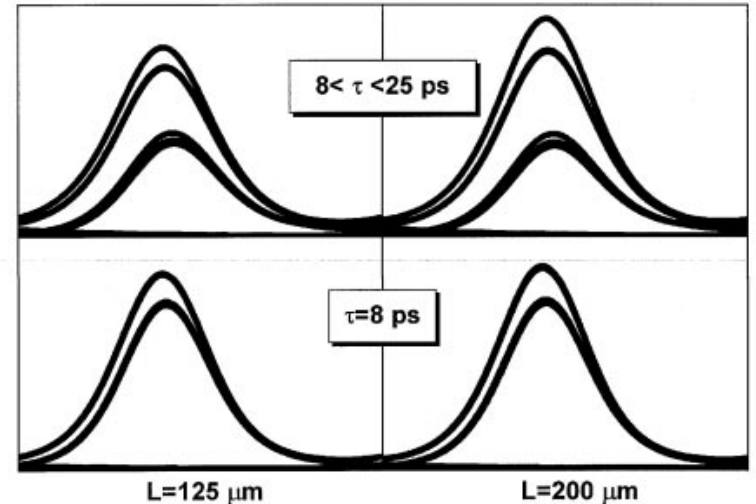

Fig. 12. Comparison of eye-diagrams for the control signal at the output of an EAM with two different functional dependences of the sweep-out time, $\tau$, on the carrier density. There is a significant reduction in the mark excursions when the sweep-out time is reduced. The results are shown for two different device lengths. The bit rate is $40 \mathrm{~Gb} / \mathrm{s}$, and the power levels were $P_{\mathrm{CW}}=10 \mathrm{dBm}$ and the average control power level was $P_{\mathrm{sig}}=19 \mathrm{dBm}$. The temporal window is $25 \mathrm{ps}$ in all cases.

the second case the sweep-out time is $8 \mathrm{ps,} \mathrm{independently} \mathrm{of}$ the carrier density. The figure shows that the reduced sweep-out time gives a substantial reduction in the excursions of the marks. Table I shows in brackets the corresponding ERs which result. Besides the reduced mark variation, also the ERs are improved significantly. This means that in this type of signal regeneration scheme it is important to keep the sweep-out time low also at higher carrier densities. The same conclusions are valid for the wavelength conversion functionality.

\section{CONCLUSION}

We have modeled wavelength conversion and signal regeneration using an EAM. The method is based on the saturable absorption characteristic of the device.

A critical dependence of the conversion efficiency on device length and input power levels was demonstrated and explained. For low bit rates $(10 \mathrm{~Gb} / \mathrm{s})$, the ER was shown to saturate at a level which increases with the control pulse input power level. We showed that for a fixed device length there is an optimum control pulse power level giving the best ER for the converted signal at the output.

We showed that the performance at up to $20 \mathrm{~Gb} / \mathrm{s}$ was quite good both for wavelength conversion and signal regeneration. Operation at $40 \mathrm{~Gb} / \mathrm{s}$, however, was significantly impaired because of serious patterning due to incomplete absorption recovery between bits. To obtain better performance, it is therefore necessary to bring down the sweep-out time. In particular, it is important to reduce the power sensitivity of the sweep-out dynamics. We demonstrated this for the case of signal regeneration, showing both a better ER and smaller mark (and space) excursions. Similar improvements take place for the converted signal.

It was shown that there can be a difference in performance between the co- and counterpropagation schemes, resulting from a less efficient carrier modulation in the co-propagation scheme. In our simulations, there was no qualitative differences in the shape of the eye-diagrams for the two schemes. 
In summary, our modeling has demonstrated that the EAM is a promising device for performing all-optical wavelength conversion and native signal regeneration. The functionality of the EAM is suitable for integration with other devices, such as an Mach-Zehnder interferometer or amplifier sections.

\section{REFERENCES}

[1] H. Soda, M. Furutsu, K. Sato, N. Okazaki, S. Yamazaki, H. Nishimoto, and $\mathrm{H}$. Ishikawa, "High-power and high-speed semi-insulating $\mathrm{BH}$ structure monolithic electroabsorption modulator/DFB laser light source," Electron. Lett., vol. 26, no. 1, pp. 9-10, 1990.

[2] M. Suzuki, H. Tanaka, K. Utaka, N. Edagawa, and Y. Matsushima, "Transform-limited $14 \mathrm{ps}$ optical pulse generation with $15 \mathrm{GHz}$ repetition rate by InGaAsP electroabsorption modulator," Electron. Lett., vol. 28, no. 11, pp. 1007-1008, 1992.

[3] K. Wakita, K. Sato, I. Kotaka, M. Yamamoto, and M. Asobe, "Transform-limited 7-ps optical pulse generation using a sinusoidally driven InGaAsP/InGaAsP strained multiple-quantum-well DFB laser/modulator monolithically integrated light source," IEEE J. Photon. Technol. Lett., vol. 5, pp. 899-901, Aug. 1993.

[4] D. G. Moodie, A. D. Ellis, and C. W. Ford, "Generation of 6.3 ps optical pulses at a $10 \mathrm{GHz}$ repetition rate using a packaged electroabsorption modulator and dispersion compensating fiber," Electron. Lett., vol. 30, no. 20 , pp. $1700-1701,1994$.

[5] M. Suzuki, H. Tanaka, and Y. Matsushima, "10 Gbit/s optical demultiplexing and switching by sinusoidally driven InGaAsP electroabsorption modulators," Electron. Lett., vol. 28, no. 10, pp. 934-935, 1992.

[6] M. Suzuki, H. Tanaka, N. Edagawa, and Y. Matsushima, "New applications of a sinusoidally driven InGaAsP electroabsorption modulators to in-line optical gates with ASE noise reduction effect," Electron. Lett., vol. 28, no. 10, pp. 934-935, 1992.

[7] S. Kawanishi, "Ultrahigh-speed optical time-division-multiplexed transmission technology based on optical signal processing," IEEE J. Quantum Electron., vol. 34, pp. 2064-2079, Nov. 1998

[8] A. D. Ellis, J. K. Lucek, D. Pitcher, D. G. Moodie, and D. Cotter, "Full $10 \times 10 \mathrm{Gbit} / \mathrm{s}$ OTDM data generation and demultiplexing using electroabsorption modulators," Electron. Lett., vol. 34, no. 18, pp. 1766-1767, 1998.

[9] B. Mason, G. A. Fish, S. P. DenBaars, and L. A. Coldren, "Widely tunable sampled grating DBR laser with integrated electroabsorption modulator," IEEE J. Photon. Technol. Lett., vol. 11, pp. 638-640, June 1999.
[10] N. Edagawa, M. Suzuki, and S. Yamamoto, "Novel wavelength converter using an electroabsorption modulator: Conversion experiments at up to $40 \mathrm{Gbit} / \mathrm{s}$," in Proc. Tech. Dig. OFC'97, vol. 6, 1997, pp. 77-78.

[11] H. Yokoyama, Y. Hashimoto, and H. Kurita, "Noise reduction in optical pulses and bit-error-rate improvement with a semiconductor-waveguide saturable absorber," in Proc. Tech. Dig. CLEO'98, vol. 6, 1998 , pp. 502-503.

[12] N. Edagawa, M. Suzuki, and S. Yamamoto, "Novel wavelength converter using an electroabsorption modulator," IEICE Trans. Electron. vol. E81-C, no. 8, pp. 1251-1257, 1998.

[13] T. Durhuus, B. Mikkelsen, C. Jørgensen, S. L. Danielsen, and K. E. Stubkjær, "All-optical wavelength conversion by semiconductor optical amplifiers," J. Lightwave Technol., vol. 14, pp. 942-954, June 1996.

[14] S. Bischoff, J. Mørk, T. Franck, S. D. Brorson, M. Hofmann, K. Fröjd, L. Prip, and M. P. Sørensen, "Monolithic colliding pulse mode-locked semiconductor lasers," Quantum Semiclass. Opt., vol. 9, pp. 655-674, 1997.

[15] J. A. Cavaillès, D. A. B. Miller, J. E. Cunningham, P. L. K. Wa, and A. Miller, "Simultaneous measurements of electron and hole sweep-out from quantum wells and modeling of photoinduced field screening dynamics," IEEE J. Quantum Electron., vol. 28, pp. 2486-2497, Oct. 1992.

[16] P. J. Bradley, C. Rigo, and A. Stano, "Carrier induced transient electric fields in a $p-i-n$ InP-InGaAs multiple-quantum-well modulator," IEEE J. Quantum Electron., vol. 32, no. 1, pp. 43-52, 1996.

[17] H. Kurita, I. Ogura, and H. Yokoyama, "Ultrafast all-optical signal processing with mode-locked semiconductor lasers," IEICE Trans. Electron., vol. E81-C, no. 2, pp. 129-139, 1998.

[18] G. P. Agrawal and N. K. Dutta, Semiconductor Lasers. New York: Van Nostrand Reinhold, 1993.

Sune Højfeldt, photograph and biography not available at the time of publication.

Svend Bischoff, photograph and biography not available at the time of publica-

Jesper Mørk, photograph and biography not available at the time of publication. 